Planetary Systems in the Universe - Observation, Formation and Evolution Proceedings IAU Symposium No. 202, (c)2004 IAU

Alan Penny, Pawel Artymowicz, Anne-Marie Lagrange, $\mathcal{G}$ Sara Russell, eds.

\title{
Bisector analysis as a diagnostic of intrinsic radial-velocity variations
}

\author{
N.C. Santos, M. Mayor, D. Naef, D. Queloz and S. Udry \\ Observatoire de Genève, CH-1290 Sauverny, Switzerland
}

\begin{abstract}
In this contribution we present the results of the application of the bisector of the cross-correlation function as a diagnostic of activity-related radial-velocity variations. The results show that the technique is very effective. We present examples for which the application of the bisector analysis was essential to establish the planetary nature of the candidate or to exclude an orbital signature. An analysis of the behaviour of the bisector for active dwarfs of different spectral types shows that the relation between the bisector and the radial-velocity variation depends in a great extent on the $v \sin i$ of the star. The results may shed a new light on the intrinsic sources of radial-velocity variation for different types of solar-type dwarfs.
\end{abstract}

\section{Introduction}

Activity-related phenomena (e.g. spots or convective inhomogeneities) can induce radial-velocity variations (Saar \& Donahue 1997; Santos et al. 2000b). These can be very important for high-precision radial-velocity planet-search programmes: the resulting radial-velocity signal can mimic a planetary orbital signature and "produce" false planetary candidates, as a result of line shape variations (e.g. bisector changes due to a rotating spot).

Here we describe the use of the bisector of the cross correlation function (as described in detail in Queloz et al. 2000) as a diagnostic of activity induced radial-velocity variations. Some examples are shown and the main results are presented and discussed.

\section{Activity vs planetary signature?}

Amongst the stars in the Geneva planet-search programmes ${ }^{1}$, some have been found to exhibit periodic radial-velocity changes correlated to bisector variations.

The standard case is HD 166435 (Queloz et al. 2000). HD 166435 is a G0 active dwarf which was found to have a periodic radial-velocity signal with a 3.8-day period and $83 \mathrm{~m} \mathrm{~s}^{-1}$ of radial-velocity semi-amplitude. Although at first the radial-velocity signal seemed to be connected to the existence of a planetary companion, the same period was observed in photometry (see Queloz et al. 2000, for further details). This lead us to study the bisector of the cross-correlation function for possible variations.

\footnotetext{
${ }^{1}$ Both ELODIE and CORALIE; see http://obswww.unige.ch/ udry/planet/planet.html
} 

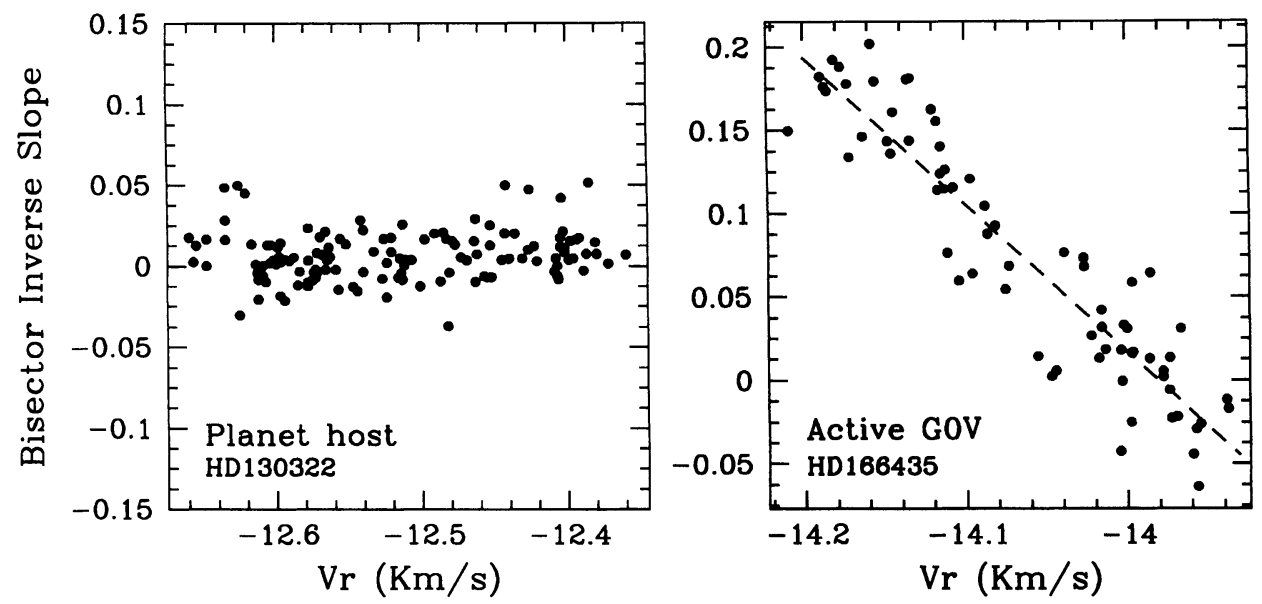

Figure 1. Bisector inverse slope as a function of the radial-velocity for two stars. Left: HD 130322, an active $\mathrm{K}$ dwarf with a planetary companion; right: HD 166435 (see text)

The results showed that the bisector inverse slope (BIS) was changing in phase with the radial-velocity signal, proving the intrinsic source of the radialvelocity variations (Fig. 1, right).

On the other hand, this technique has been successfully applied to confirm the presence of planetary companions to the solar-type active dwarfs HD 192263 (Santos et al. 2000a), HD 130322 (Udry et al. 2000) and HD 1237 (Naef et al. 2000a, 2000b), all K dwarfs. The case of HD 130322 can be seen in Fig. 1 (left). No trend is observed, confirming that the radial-velocity signal is not related to changes in the bisector of the cross-correlation function.

\section{Active stars and bisector behaviour}

We have analyzed a sample of 14 active $\mathrm{G}$ and $\mathrm{K}$ dwarfs (defined here as having $\log R_{H K}^{\prime}>-4.4$ ) from the CORALIE and ELODIE planet-search samples, having more than $\sim 20$ high-precision radial-velocity measurements. For those stars we have computed the bisector inverse slope to be compared to the radialvelocity values.

To test a possible relation with spectral type we have plotted in Fig. 2 (left) the values of the slope of the relation BIS vs. RV against the colour index $B-V$. The observed trend seems to suggest that although active late $F$ and early $G$ dwarfs do induce radial-velocity changes by altering the line profiles, later type active dwarfs are less sensitive to such line asymmetries. That may explain why this latter objects have, for a given activity level, lower radial-velocity "jitter" (Santos et al. 2000b). However, as it is clear from Fig. 2 (right), this effect is in great extent controlled by the $v \sin i$ of the star. More data at a given $v \sin i$ is needed to unveil other possible dependences e.g. with colour or activity level. Stars with planetary companions that were included after subtraction of the orbital solution do appear in the main trend. 

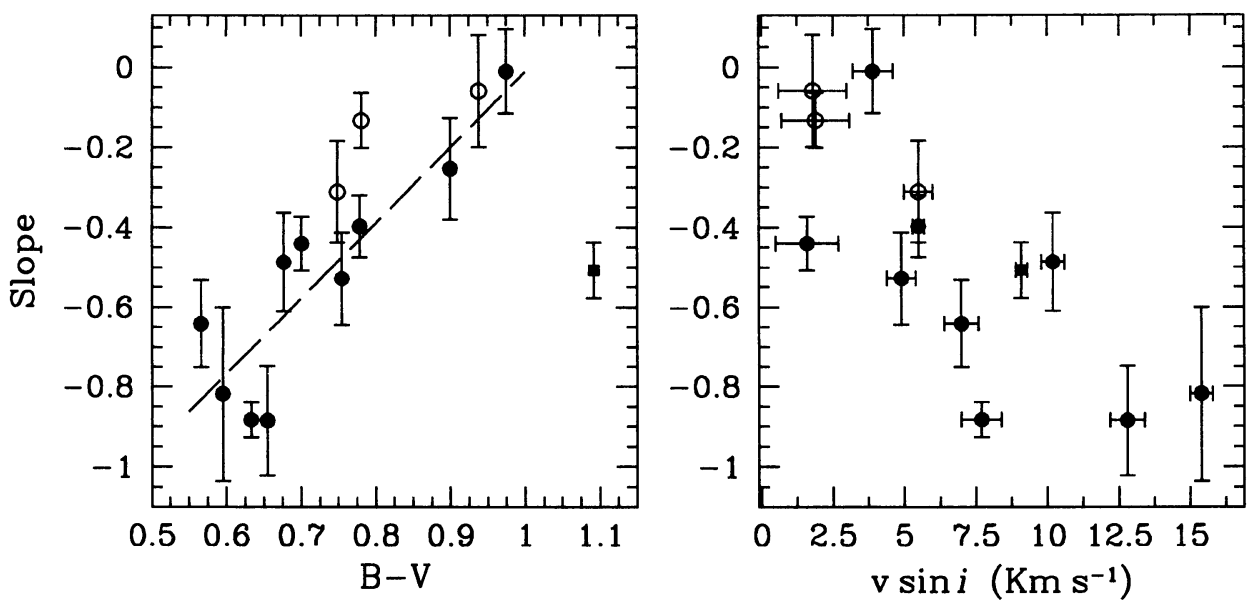

Figure 2. Left: Slope of the relation BIS vs. RV plotted against $B-V$. The best linear non-weighted fit to the points with $(B-V)<1$ is presented. Active stars with planets (open dots) are plotted after subtraction of the orbital signal. The square corresponds to a K3 dwarf with a particularly high chromospheric activity index (HD 29697, $\log R_{H K}^{\prime} \sim-4.1$; Duncan et al. 1991) and $v \sin i$; it is not included in the fit. Right: Values of the same Slope against $v \sin i$

A better knowledge of the relations of Fig. 2 may became useful in the future, since it may represent a way of disentangling between activity-induced and orbital radial-velocity variations.

\section{References}

Duncan, D.K., Vaughan, A.H., Wilson, O.C., et al. 1991, ApJ, 76, 383

Naef, D., Mayor, M., Pepe, F., Queloz, D., Santos, N., Udry, S., \& Burnet, M. 2000a. In: "Disks, Planetesimals and Planets", Garzon, F., Eiroa, C., de Winter, D., \& Mahoney, T.J., ASP Conference Series, in press

Naef, D., Mayor, M., Pepe, F., Queloz, D., Santos, N., Udry, S., \& Burnet, M. 2000b, $\mathrm{A} \& \mathrm{~A}$, in press

Queloz, D., et al. 2000, in prep.

Saar, S.H., \& Donahue, R.A. 1997, ApJ, 485, 319

Santos, N.C., Mayor, M., Naef, D., Pepe, F., Queloz, D., Udry, S., Burnet, M., \& Revaz, Y. 2000a, A\&A, 356, 599

Santos, N.C., Mayor, M., Naef, D., Pepe, F., Queloz, D., Udry, S., \& Blecha, A. 2000b, A\&A, 361, 265

Udry, S., Mayor, M., Naef, D., Pepe, F., Queloz, D., Santos, N.C., Burnet, M., Confino, B., \& Melo, C. 2000, A\&A, 356, 590 